Sportwiss $2013 \cdot 43: 56-57$

DOI 10.1007/s12662-013-0289-5

Online publiziert: 20. März 2013

๑) Springer-Verlag Berlin Heidelberg 2013
Wolfgang I. Schöllhorn · Hendrik Beckmann · Alexander Eekhoff · Patrick Hegen

Trainings- und Bewegungswissenschaft, Johannes Gutenberg Universität, Mainz, Deutschland

\title{
Differenzielles Lehren und Lernen: (k)eine Kritik - eine Bestätigung
}

\author{
Wahrheit ist die Erfindung eines \\ Lügners (H. v. Förster)
}

\section{Diskussionsbeitrag zu}

Künzell, S. \& Hossner, E.-J. (2012).

Differenzielles Lehren und Lernen: eine Kritik. Sportwissenschaft, 42(2), 83-95

Wir, die Autoren, antworten an dieser Stelle auf o.g. Beitrag. Redaktionelle Gründe zwingen uns zur Beschränkung auf die Reflektion wesentlicher Vorwürfe gegenüber dem differenziellen Lernen und Lehren (DL) und den Hinweis auf ein fragwürdiges Wissenschaftsverständnis in jenem Artikel. Eine ausführliche Darstellung des differenziellen Lehr- und Lernansatzes mit Grundlagen und Abgrenzungen wird daher an anderer Stelle erfolgen. Wir freuen uns über jede wissenschaftliche Diskussion und nehmen diese Gegendarstellung gerne zum Anlass, wesentliche inhaltliche Fehldarstellungen in o.g. Artikel aufzuzeigen und zu korrigieren.

Aus wissenschaftstheoretischer Sicht sind generalisierbare Strukturen von Klassen von Tatsachen und allgemeine Beziehungen zwischen Klassen und Tatsachen Gegenstand der Wissenschaft, die auf der Basis unterschiedlichster Theorien interpretiert werden können (Detel, 2008). Zwar verweist die Kritik implizit auf diesen Pluralismus, indem zur Erklärung des Kontextinterferenz(CI)-Paradigmas unterschiedliche kognitivistische Ansätze akzeptiert werden, lehnt ihn jedoch ab, wenn das Erklärungsmodell des DL das CI-Paradigma aus systemdynami- scher Sicht erklärt und integriert. Hierbei wird implizit auf das Einfachheitsprinzip („Occam's razor") verwiesen, indem u. a. der Elaborationshypothese oder vertieften Verarbeitung, trotz der Widersprüche bei komplexen Bewegungsaufgaben und beim Bewegungslernen von Kindern, die wesentlich einfachere Erklärung für die Vorteile randomisierten Übens zugeschrieben wird. Das gleiche Prinzip wird jedoch abgelehnt, wenn die Grundlagen des DL über den Erklärungsbereich des CI-Lernens hinausgehen und die Widersprüche inklusive anderer Lerntheorien miterklären. Die damit verbundene Suggestion der Existenz einer einzigen Wahrheit erscheint uns nicht mehr ganz zeitgemäß. Ein ähnlich monistisches Wissenschaftsbild scheint auch Pate zu stehen, wenn auf der einen Seite dem DL nichts Neues im Vergleich zu CI-Lernen und keine Praktikabilität zugeschrieben wird, gleichzeitig jedoch beim CI-Lernen eine große Praxisrelevanz suggeriert wird. Unabhängig von den logischen Widersprüchen dieser Gedankenkonstruktion schreiben Übersichtsartikel dem CILernen bislang wenig praktische Relevanz $\mathrm{zu}$ und die praxisbezogene Rezeption ist auffallend gering. Stringent lässt sich das widersprüchliche Wissenschaftsverständnis auch im Umgang mit den statistischen Untersuchungsmethoden beobachten. Werden Ergebnisse ältere Modelle auf der Basis der gleichen Untersuchungsmethodik (Fischer-Statistik) nahezu kritiklos übernommen, wird von den Untersu- chungen zum DL eine modischere Untersuchungsmethode (Neyman-Pearson-Statistik) erwartet, die bereits schon wieder in der Kritik steht (Hurlbert \& Lombardi, 2009). Sämtliche Untersuchungen zum DL wurden bislang auf der Basis der gleichen Methodik wie bislang bekannte Modelle zum motorischen Adaptationsprozess durchgeführt.

Eine ähnliche Problematik des Wissenschaftsverständnisses zeigt sich beim Umgang der Kritik mit dem Erkenntnisprozess durch Deduktion und Induktion. Wird eine in einem früheren Prozess generalisierte Klasse (Dynamik dissipativer Systeme) anhand eines Beispiels (Modell des Phasenübergang bei zyklischen Fingerbewegungen) und weiteren Vereinfachungen (Modell des Potenzials) metaphorisch erläutert (Schöllhorn, 1999), dieses eine Beispiel dann jedoch aufgegriffen, um andere Aspekte der Vereinfachung, ungeachtet des Verkürzungsmerkmals und der fehlerhaften Wiedergabe als generalisierte Struktur darzustellen [u. a. In-phase und Anti-phase sind im Vergleich zur gängigen Literatur vertauscht, am Phänomen orientiert und nicht an der Kontrolle (1), die Potenzialmulde der Inphase-Bewegung wird bei zunehmender Frequenz nicht tiefer (2)], dann leidet das wissenschaftliche Verständnis von deduktivem und induktivem Erkenntnisprozess. Bleiben wir im allgemeineren (abstrakteren) Bereich der Dynamik dissipativer Systeme, so kommt dort Fluktuationen und ihrer Zunahme bei Phasenübergän- 
gen eine zentrale Rolle zu. Die Steigerung der Fluktuationen wird beim DL widerspruchsfrei zum einen von der beschreibenden Ebene auf die Interventionsebene und zum anderen analog zahlreichen Beispielen aus der Technik und Neurobiologie (stochastische Resonanz) auf die Verhaltens-/Lernebene übertragen, d. h. DL ist aus den Grundlagen der Systemdynamik ableitbar.

Die Problematik setzt sich fort bei der Interpretation des Umgangs mit Rückinformation, indem von der Aussage, dass beim DL vom Trainer keine externe Rückinformation an den Athleten geliefert wird, durch die Kritiker auf eine generelle Unterbindung von Rückinformation geschlossen wird und damit Rückmeldeinformationen des Athleten selbst gleichzeitig mitunterbunden werden. Auch einschlägige Literatur liefert Hinweise dafür, dass externe Rückinformation in erster Linie dann notwendig ist, wenn der Athlet nicht in der Lage ist, neue Informationen aus der Bewegungsausführung zu extrahieren bzw. es selbst nicht spürt, (was bei einer versuchten Wiederholung auch schwierig scheint, da ja versucht wurde das Gleiche zu reproduzieren). Bei größeren Differenzen aufeinanderfolgender Bewegungen sind zusätzliche externe Rückmeldeinformationen redundant. Zusammenfassend: 1) Das DL ist aus dem systemdynamischen Ansatz ableitbar. 2) Eine Abgrenzung des DL zum Kognitivismus war nie vorgesehen. 3) Die Effekte des DL sind empirisch bestätigt 4) Die nationale und internationale Rezeption in der Praxis bestätigt den großen Praxisbezug des Neuen am DL.

Bleibt zu hoffen, dass die zahlreichen offenen Fragestellungen zum differenziellen Lehren und Lernen zukünftig zu umfangreichen konstruktiven Diskussionen und Experimenten aus unterschiedlichster Perspektive mit weniger Widersprüchen führen.

\section{Korrespondenzadresse}

\section{Prof. Dr. W. I. Schöllhorn}

Trainings- und Bewegungswissenschaft

Johannes Gutenberg Universität

Albert Schweitzer Straße 22, 55099 Mainz

move.brain@uni-mainz.de

\section{Literatur}

Detel, W. (2008). Grundkurs Philosophie, Band 4, Erkenntnis- und Wissenschaftstheorie. Stuttgart: Reclam.

Hurlbert, S. H., \& Lombardi, C. M. (2009). Final Collapse of the Neyman-Pearson Decision Theoretic Framework and Rise of the neoFisherian. Annales Zoologici Fennici, 46(5), 311-349.

Schöllhorn, W. I. (1999). Individualität - ein vernachlässigter Parameter? Leistungssport, 29(2), 7-11. 